\title{
Nanofood for thought
}

\author{
The food industry will only reap the benefits of nanotechnology if issues related to safety are addressed \\ and companies are more open about what they are doing.
}

There are many reasons to object to the UK's House of Lords, such as the fact that it is unelected, but its critics must also admit that some of the finest minds in the UK are members of the Lords and its committees. This means that when the Lords' Science and Technology Committee, for example, focuses its attention on a topic such as nanotechnologies and food, it can call on the likes of John Krebs, the noted zoologist and former chairman of the UK's Food Standards Agency (FSA), and Robert May, the noted ecologist and former chief scientific advisor to the UK government. The committee's report on this topic ${ }^{1}$, published last month, is a valuable addition to discussions on the impact of nanomaterials on human health, even though the committee acknowledges that many of its 32 recommendations - which cover commercialization, health and safety, the definition of nanomaterials, regulation and communication - echo those of previous reports on nanotechnology ${ }^{2}$.

\section{There are relatively few foods containing engineered nanomaterials on the market at present.}

So far nanotechnology has largely escaped becoming 'the next GM' - which is shorthand for the rejection of genetically modified food by the public in the UK and elsewhere in Europe - but this has largely been because many applications of nanotechnology have been inherently non-controversial: who can object to stain-free trousers or faster computers? The popularity of products such as the iPod Nano has also helped with public acceptance of nanotechnology, even to the extent that distinctly non-nano products such as the Tata Nano car - have sought to exploit the 'nano' brand. However, the introduction of nanomaterials into food and food packaging is a completely different matter, involving important factors that do not arise when developing new materials or electronic devices.

Nanotechnology could benefit the food industry and consumers in two main ways $^{3}$ : by using engineered nanomaterials to reduce the amount of fat, salt or sugar in food without changing its taste; and by developing new packaging that keeps food fresher for longer and, possibly, tells the consumer if the food inside has gone off. Improved packaging might also allow more foods to be stored under ambient conditions, rather than in fridges and freezers, thus reducing energy consumption.

\section{The committee does not support the idea of compulsory labels for nanofoods.}

However, as the Lords' report makes clear, there are relatively few foods containing engineered nanomaterials on the market at present. Indeed, the FSA told the committee that it was aware of just two food products containing nanomaterials - both food supplements on the market in the UK. Moreover, one of these (a nanoscale form of silver called silver hydrosol) is likely to be banned by the European Food Safety Authority in the near future because there is not enough information available to determine its potential effects on the human body.

At the same time, it is estimated that up to 400 companies around the world are researching possible applications of nanotechnology in food and food packaging - and many of them don't want their customers to know about this. The committee says that it is "regrettable" that "far from being transparent about its activities, the food industry was refusing to talk about its work in this area." While acknowledging that the food industry is afraid that the public might react negatively to food and food packaging that contains engineered nanomaterials, the committee argues that "this is exactly the type of behaviour which may bring about the public reaction which it is trying to avert."

In its most radical recommendation the committee calls for the FSA, in collaboration with the food industry, to set up a confidential database of information about research into nanomaterials being carried out in the food sector. This information would be used in the development of new approaches to risk assessment, and would also help to set priorities for research. Significantly, participation in the database would be mandatory for companies. However, the committee does not support the idea of compulsory labels for nanofoods, recommending instead a public register of foods that contain nanomaterials.

The recommendations on the commercialization of nanotechnologies in the food sector - such as collaborations between industry, universities and others on precompetitive research, or better knowledge transfer - will be familiar to anyone with even a passing interest in science policy in the UK over the past two decades. There is also a familiar ring to the recommendations that call for more research into the impact of nanomaterials on the human body, but the committee is more specific about what needs to be done, the gaps and grey areas in current knowledge, the benefits of coordinating research at an international level to avoid unnecessary duplication of effort, and the need to build capacity for such research. Somewhat alarmingly, the report reveals that there is just one research group in the UK studying how nanomaterials interact with the gastrointestinal tract, although Krebs told this journal that four to five groups would be enough to provide the capacity needed. The committee is also to be commended for stressing that any definition of a nanomaterial needs to be based on evidence for behaviour that is different from that seen in the bulk, rather than some arbitrary size such as $100 \mathrm{~nm}$ (refs 4,5).

Nanotechnology has much to offer to the food industry, and this report has much to offer food manufacturers, government, funding agencies and regulators. They are advised to digest it carefully.

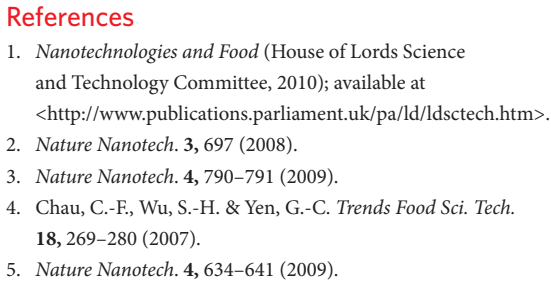

